\title{
Cambios en las condiciones físicas, químicas y faunísticas de un sistema fluvial (río Oma, Bizkaia), y desaparición de una población de cangrejo autóctono (Austropotamobius pallipes): ¿causa y/o efecto?
}

\author{
A. Rallo*, L. García-Arberas y A. Antón \\ Laboratorio de Zoología. Universidad del País Vasco. Apdo. 644. 48080 Bilbao, Spain \\ *zopragra@lg.ehu.es
}

\begin{abstract}
RESUMEN
El Oma (cuenca del río Oka, provincia de Bizkaia) es un pequeño río relativamente poco alterado que recorre terrenos de uso forestal, agrícola y ganadero. En él habitaba una población bien establecida de cangrejo autóctono Austropotamobius pallipes, pero ésta había desaparecido en el verano de 1995, sin que se hubieran registrado mortandades masivas como las características de un episodio de afanomicosis. En este trabajo se estudia la evolución de las condiciones fisicoquímicas y de las comunidades bentónicas estivales del río Oma desde el año 1994 hasta el 2001, comparado esta dinámica respecto a las condiciones generales que presentan los hábitats de otras poblaciones de cangrejo autóctono en la red fluvial del entorno. Aparte de esta desaparición del cangrejo, también se han detectado cambios en algunas condiciones del agua así como en la composición faunística de la comunidad de macroinvertebrados. Se describen estos cambios temporales y se discuten sus posibles causas, especialmente la que ha generado la extinción de la especie protegida (influencia de depredadores, pérdida de calidad de hábitat por vertidos o lixiviados, intervenciones en cauce), pero aunque en otras cuencas cercanas se ha podido encontrar la causa principal de mortandad y/o desaparición de cangrejos, no así en el Oma, en donde podría haber tenido lugar una sinergia entre varios factores. No obstante, la evolución temporal del sistema río en los últimos años parece presentar una tendencia a recuperar las condiciones que tenía en el año 1994, tanto en la fisicoquímica como en la composición faunística.
\end{abstract}

Palabras clave: Austropotamobius pallipes, sistema fluvial, fisicoquímica del agua, macroinvertebrados bentónicos, Bizkaia, España

\begin{abstract}
The Oma (Oka basin, province of Biscay) is a relatively little perturbed river, which runs through places where the main activities are forestry, agriculture and cattle raising. The native crayfish Austropotamobius pallipes was well established on it but in the summer of 1995 this population had disappeared. Crayfish mass mortalities that could have been due to the crayfish plague were not detected. The main objective of this work is to study the changes of abiotic and biotic summer conditions of the Oma River from 1994 to 2001. The Oma River conditions are compared with the general conditions of the close fluvial reaches inhabited by native crayfish. In addition to the disappearance of the crayfish population, changes in water chemistry and faunal composition of the benthic community were detected. Temporal variations are described in this work and the possible causes are also discussed, especially those related with the extinction of the protected species (predators' influence, decrease of the habitat quality by leaches, works on the riverbed).

We have been able to detect the main cause for the mortality and/or disappearance of crayfish in other close fluvial zones, but not in the Oma River. In this stream it could have been due to the synergic interaction of several factors. However, the temporal evolution of the river system in the last years seems to show a trend to recover the conditions of the river on both physicochemical quality and faunal composition, that had in 1994.
\end{abstract}

Keywords: Austropotamobius pallipes, fluvial system, water chemistry, benthic macroinvertebrates, Biscay, Spain.

\section{INTRODUCCIÓN}

El río Oma, localizado en la Reserva de la Biosfera de Urdaibai en la provincia de Bizkaia, es un pequeño curso fluvial relativamente muy poco alterado que recorre terrenos de uso forestal, agrícola y ganadero. La existencia de cangrejo autóctono Austropotamobius pallipes en este 
río era conocida desde tiempo inmemorial, si bien la especie pudo ser introducida por los habitantes de la zona. Posiblemente por su aislamiento no se vio afectada por la afanomicosis que asoló los ríos de Bizkaia a finales de los años 70 .

En el verano de 1994 se inició una investigación para localizar en la red fluvial de Bizkaia, incluido el río Oma, los tramos fluviales donde se encontraran poblaciones de la especie de cangrejo nativo, A. pallipes, y evaluar su demografía $\mathrm{y}$ condiciones del hábitat. Se encontró en el Oma, pero en la siguiente campaña de muestreo, en 1995, esta población había desaparecido totalmente. No había habido mortandades masivas como las características de un episodio de afanomicosis (Söderhäll \& Cerenius, 1999), que sin duda habrían sido detectadas por la Guardería del Servicio de Montes de la Diputación, pero en ese año, y especialmente en el siguiente, sí se encontraron cambios importantes en algunas de las condiciones del río. Todo ello motivó un proyecto particular de estudio de las condiciones abióticas y bióticas del río en ese y sucesivos años para medir su cambio temporal, determinar las posibles causas de la desaparición del cangrejo, así como evaluar la recuperación de su hábitat en relación con la posibilidad de la reaparición de la especie, bien de forma natural o por reintroducción controlada.

En este trabajo se presentan los resultados correspondientes a la evolución de estas condiciones desde 1994 hasta el 2001, y se comparan las características físico-químicas del agua del Oma respecto a las de otros arroyos de la red fluvial del entorno en los que sí se han encontrado poblaciones de cangrejo. Se discuten las posibles causas de esta perturbación en el sistema río que hayan llevado a la desaparición del cangrejo, así como la influencia de ésta en el sistema.

\section{MATERIAL Y MÉTODOS}

La pequeña subcuenca del Oma pertenece a la cuenca del río Oka en el Territorio Histórico de Bizkaia (Fig. 1). El cauce principal de la subcuenca es de primer orden y casi único salvo

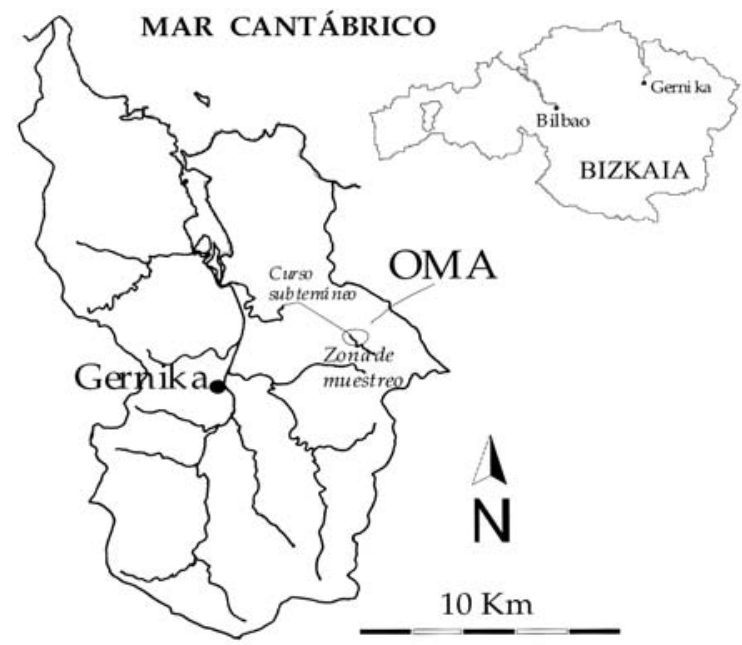

Figura 1. Localización del río Oma, perteneciente a la cuenca del Oka, en la provincia de Bizkaia, en el norte de la Península Ibérica. Location of the Oma River, in the Oka basin, in the province of Biscay, northern Iberian Peninsula.

pequeños arroyos afluentes. Tiene casi $3 \mathrm{~km}$. de longitud y recoge las aguas de una zona cárstica (cuenca de recepción de unos $25 \mathrm{~km}^{2}$ ) y, tras desaparecer en una dolina, emerge posteriormente en el manantial de Olalde, desembocando en la margen derecha del estuario de Gernika. En el tramo antes del sumidero el cauce se halla relativamente encajado a profundidades de $0.5 \mathrm{a}$ $1 \mathrm{~m}$, con un perfil de elevada pendiente, como consecuencia del aterrazamiento provocado por los cultivos adyacentes. La anchura de cauce se mantiene constante a lo largo del recorrido en torno a $1.5-2 \mathrm{~m}$, con un valor máximo ligeramente superior a $2.5 \mathrm{~m}$ cerca del sumidero. El lecho del río está compuesto principalmente de piedras pequeñas y gravas. En este trayecto predominan las zonas de rápidos y las pozas que se forman no tienen gran profundidad. El agua discurre sin saltos ni discontinuidades importantes.

Los cultivos adyacentes están formados principalmente por prados que se siegan hasta las márgenes, protegiéndoles frente a una elevada erosión pero proporcionando poca sombra al cauce. Se observa también en esta área algunas manchas de matorral y zarzas aisladas junto a árboles solitarios situados junto al cauce. Aguas 
arriba predominan los cultivos forestales de pino y se reducen progresivamente los prados. La vegetación de la cuenca se completa con zonas de encinar cantábrico bien conservadas.

El tramo de río estudiado se situó a su paso por el barrio de Oma, a unos $3 \mathrm{Km}$. del origen y unos $100 \mathrm{~m}$ antes de desaparecer en la dolina (U.T.M. 30TWN31279833). El estudio se llevó a cabo con carácter anual durante los veranos de los años 1994 a 2001 (excepto en 1999). El muestreo de cangrejos se realizó siguiendo la metodología habitual para este tipo de pequeños arroyos en los que no es posible emplear nasas: los ejemplares se capturan a mano después del anochecer, utilizando una linterna frontal, durante un tiempo determinado (30 minutos en el caso del Oma), refiriéndose los datos a esfuerzo de captura (García-Arberas \& Rallo, 2000).

Para la caracterización del hábitat en el tramo fluvial se toman datos de anchura de cauce (húmedo y seco), profundidad y velocidad de la corriente (mediante un correntímetro Swoffer Instruments, modelo 2100). A partir de estos valores se calcula el caudal instantáneo. También se miden en campo la temperatura del aire y agua, el pH (pHmetro WTW, modelo 320), la conductividad (conductivímetro WTW, modelo LF320) y el oxígeno disuelto (oxímetro WTW, modelo 320), y se toma una muestra de agua para su posterior análisis en laboratorios homologados utilizando metodología estándar (APHAAWWA-WPCF, 1998). Se midieron las siguientes variables: dureza, $\mathrm{Cl}^{-}, \mathrm{SO}_{4}^{-2}, \mathrm{HCO}_{3}^{-}, \mathrm{Na}^{+}, \mathrm{K}^{+}$, $\mathrm{Mg}^{+2}, \mathrm{SiO}_{2}, \mathrm{Ca}^{+2}, \mathrm{NO}_{3}^{-}, \mathrm{NO}_{2}^{-}, \mathrm{NH}_{4}^{+}$, fósforo total y sólidos en disolución y en suspensión.

La fauna de macroinvertebrados bentónicos se estudia a partir de muestras tomadas mediante red Kick, que se limpiaban in situ $(1 \mathrm{~mm}$ de luz de malla). La identificación taxonómica se realiza al nivel preciso para el cálculo de los índices bióticos y de comunidad (abundancia, dominancia, diversidad de Shannon - $\log _{2}$, sobre la diversidad a nivel de familia). La calidad del agua se evaluó mediante el índice IBMWP (Alba-Tercedor \& Sánchez-Ortega, 1988), que resulta ser el más adecuado para los ríos vascos (Rico et al., 1992).
Los valores de las variables fisicoquímicas del agua se han sometido a análisis de estadística descriptiva y multivariante. También se ha realizado un análisis de ordenación (factorial de correspondencias), a partir de la matriz de abundancias de macroinvertebrados (previa transformación logarítmica). El estudio conjunto de fauna y química del agua se realiza mediante análisis de correlación de Pearson.

En un trabajo previo (Rallo \& García-Arberas, 2002) se había establecido la relación entre la distribución geográfica de las especies de cangrejo autóctono e introducido (A. pallipes y Pacifastacus leniusculus) habitantes en diversos tramos de ríos de Bizkaia con las condiciones fisicoquímicas del agua, proponiéndose un modelo descriptivo que discriminaba dentro de los tramos potencialmente aptos para la vida de los cangrejos los habitados o no por una u otra especie. Para la construcción de este modelo se utilizaron 155 datos, entre los que se encuentran los correspondientes al río Oma desde el inicio del estudio hasta 1998. En el presente trabajo se aplica el modelo de forma predictiva para las muestras de agua del Oma del 2000 y 2001.

Para el tratamiento de los datos se han utilizado los programas NTSYS-PC vers. 2.0 (Rohlf, 1997) y Statgraphics Plus vers.4.1. (Manugistics Inc., 1997).

\section{RESULTADOS}

En el tramo de estudio se encontró una población de cangrejo autóctono en la campaña de 1994. Se capturaron 99 ejemplares en 30 minutos $(2$ muestreadores en un tramo de unos $25 \mathrm{~m}$ ), lo que supone un rendimiento de captura muy elevado en comparación con otras zonas de la red fluvial de Bizkaia (García-Arberas \& Rallo, 2000). En las campañas siguientes no se ha recogido ningún ejemplar, a pesar de haber ampliado considerablemente la longitud del tramo y el esfuerzo de muestreo. Esta población habitaba un tramo fluvial cuyas condiciones abióticas y bióticas (comunidad de macroinvertebrados) se estudiaron desde 1994 al 2001. 
Tabla 1. Estadística descriptiva de las variables medidas en el agua del río Oma, a lo largo de 7 campañas de muestreo, entre los años 1994 y 2001. (FOSF $=$ fósforo total; SDIS = sólidos disueltos; SSUS = sólidos en suspensión). Summary statistics of the estimated variables in the Oma River, during the 7 sampling seasons, from 1994 to 2001. (FOSF=total phosphorus; SDIS = dissolved solids; SSUS = suspended solids).

\begin{tabular}{|c|c|c|c|c|c|c|c|c|c|c|}
\hline & $\underset{{ }^{\circ} \mathbf{C}}{\text { Temp }}$ & pH & $\begin{array}{l}\text { Cond } \\
\mu \mathrm{S} / \mathrm{cm}\end{array}$ & $\begin{array}{c}\mathrm{O}_{2} \\
\mathrm{mg} / \mathrm{l}\end{array}$ & $\begin{array}{c}\text { FOSF } \\
\text { mg/l }\end{array}$ & $\begin{array}{l}\mathrm{NO}_{3}^{-} \\
\mathrm{mg} / \mathrm{l}\end{array}$ & $\begin{array}{l}\mathrm{NO}_{2}^{-} \\
\mathrm{mg} / \mathrm{l}\end{array}$ & $\begin{array}{c}\mathrm{NH}_{4}^{+} \\
\mathrm{mg} / \mathrm{l}\end{array}$ & $\begin{array}{l}\text { SDIS } \\
\mathrm{mg} / \mathrm{l}\end{array}$ & $\begin{array}{c}\text { SSUS } \\
\mathrm{mg} / \mathrm{l}\end{array}$ \\
\hline $\mathrm{N}^{\mathrm{o}}$ muestras & 7 & 7 & 7 & 5 & 7 & 7 & 7 & 7 & 5 & 4 \\
\hline Media & 18.2 & 8.4 & 326.4 & 9.0 & 0.119 & 5.413 & 0.031 & 0.151 & 196.0 & 99.5 \\
\hline Desviación estándar & 3.4 & 0.3 & 161.5 & 1.6 & 0.087 & 0.934 & 0.008 & $3 \quad 0.260$ & 23.9 & 19.7 \\
\hline Error estándar & 1.3 & 0.1 & 61.1 & 0.7 & 0.033 & 0.353 & 0.003 & 0.098 & 10.7 & 9.8 \\
\hline Mínimo & 14.4 & 7.9 & 82.4 & 7.6 & 0.015 & 4.410 & 0.015 & 0.010 & 172.0 & 82.0 \\
\hline Máximo & 24.0 & 8.9 & 446.0 & 11.4 & 0.220 & 7.110 & 0.040 & 0.730 & $230.0 \quad 1$ & 122.0 \\
\hline Sesgo estándar & 0.750 & 0.246 & -1.265 & 0.919 & -0.157 & 1.008 & -1.298 & 2.677 & 0.617 & 0.247 \\
\hline Kurtosis estándar & 0.014 & 0.135 & -0.468 & -0.302 & -1.150 & 0.454 & 1.345 & 3.394 & -0.490 & -1.709 \\
\hline \multirow[t]{2}{*}{ Coeficiente de variación (\%) } & 18.7 & 3.5 & 49.5 & 17.8 & 73.1 & 17.3 & 26.2 & 172.2 & 12.2 & 19.8 \\
\hline & $\begin{array}{c}\text { Dureza } \\
{ }^{\circ} \mathbf{F}\end{array}$ & $\underset{\mathrm{mg} / \mathrm{l}}{\mathrm{Cl}^{-}}$ & $\underset{\mathrm{mg} / \mathrm{l}}{\mathrm{HCO}_{3}^{-}}$ & $\begin{array}{l}\mathrm{Ca}^{+2} \\
\mathrm{mg} / \mathrm{l}\end{array}$ & $\begin{array}{c}\mathrm{Mg}^{+2} \\
\mathrm{mg} / \mathrm{l}\end{array}$ & $\begin{array}{l}\mathrm{Na}^{+} \\
\mathrm{mg} / \mathrm{l}\end{array}$ & $\begin{array}{c}\mathrm{K}^{+} \\
\mathrm{mg} / \mathrm{l}\end{array}$ & $\begin{array}{l}\mathrm{SiO}_{2} \\
\mathrm{mg} / \mathrm{l}\end{array}$ & $\begin{array}{c}\mathrm{SO}_{4}^{-2} \\
\mathrm{mg} / \mathrm{l}\end{array}$ & $\begin{array}{c}\text { caudal } \\
\text { 1/sg }\end{array}$ \\
\hline $\mathrm{N}^{\circ}$ muestras & 7 & 7 & 7 & 7 & 7 & 7 & 7 & 7 & 7 & 5 \\
\hline Media & 16.0 & 15.7 & 138.5 & 54.4 & 5.8 & 10.1 & 3.6 & 4.7 & 25.8 & 28 \\
\hline Desviación estándar & 3.2 & 2.8 & 29.8 & 13.4 & 1.5 & 2.8 & 0.9 & 1.4 & 3.7 & 9 \\
\hline Error estándar & 1.2 & 1.1 & 11.3 & 5.1 & 0.6 & 1.0 & 0.3 & 0.5 & 1.4 & 4 \\
\hline Mínimo & 13.0 & 12.8 & 98.0 & 43.6 & 4.8 & 5.7 & 2.1 & 2.7 & 21.8 & 14 \\
\hline Máximo & 22.9 & 21.1 & 173.0 & 83.4 & 9.0 & 14.4 & 5.2 & 6.2 & 32.0 & 39 \\
\hline Sesgo estándar & 2.281 & 1.395 & -0.351 & 2.305 & 2.214 & -0.052 & 0.316 & -0.750 & 0.769 & -0.569 \\
\hline Kurtosis estándar & 2.731 & 1.034 & -0.886 & 2.748 & 2.188 & 0.154 & 1.527 & -0.682 & -0.280 & 0.559 \\
\hline Coeficiente de variación (\%) & 20.1 & 17.8 & 21.5 & 24.6 & 26.5 & 27.4 & 24.6 & 29.1 & 14.3 & 33.0 \\
\hline
\end{tabular}

La estadística descriptiva de las variables físicoquímicas medidas en el agua del río se presenta en la Tabla 1. Las variables más homogéneas, con un coeficiente de variación (C.V.) inferior a un $20 \%$, resultaron ser el $\mathrm{pH}$, los sólidos en disolución, las concentraciones de $\mathrm{SO}_{4}^{-2}, \mathrm{NO}_{3}^{-} \mathrm{y} \mathrm{Cl}^{-}$, el oxígeno, la temperatura y los sólidos en suspensión. El resto de las variables se consideran homogéneas (C.V. $<25 \%)$ o algo heterogéneas $(25 \%<\mathrm{C} . \mathrm{V}$. $<60 \%)$, excepto la concentración de $\mathrm{NH}_{4}{ }^{+}$, variable muy heterogénea. La variación temporal de algunas de ellas se ha representado en la figura 2. Los cambios más acusados se observan en los valores de conductividad y en menor medida del sodio. En el resto de campañas se registran incrementos puntuales de algunas variables. Según el conjunto de datos fisicoquímicos la evolución temporal de las condiciones de las muestras de agua muestra un primer grupo de mayor semejanza, correspon- diente a los años 1994 y 1995, del que se diferencian los años siguientes con una vuelta hacia las condiciones iniciales del estudio detectadas en la muestra del último año 2001 (Fig. 3).

En todos los años de estudio, en el río Oma se han identificado 52 familias o taxones de rango superior en las muestras de macroinvertebrados bentónicos recogidas (Tabla 2), de las que 18 aparecen en todas o casi todas ellas, aunque con valores de abundancia -total y relativa (dominancia)- muy distintos. Las familias que presentaban una dominancia media (calculada a partir de su abundancia relativa en cada una de las campañas como \% \pm desviación estándar) más elevada para todo el estudio fueron: Hydrobiidae $(51.9 \pm 25.1)$, los efemerópteros Baetidae $(11.0 \pm 9.5)$ y Ephemerellidae (5.4 \pm 5.2), Chironomidae $(7.8 \pm 4.6)$, coleópteros de las familias Elmidae $(5.9 \pm 2.87)$ y Helodidae $(2.6 \pm 6.0)$ y los oligoquetos $(2.4 \pm 2.6)$. 

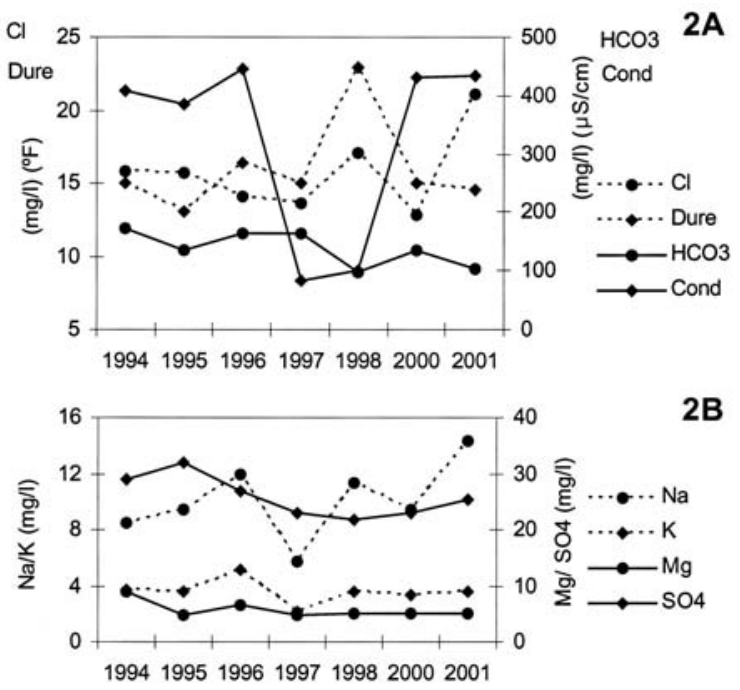

2B

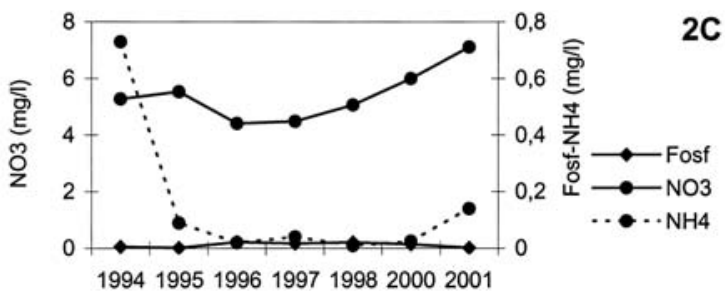

Figura 2. Evolución temporal de algunas de las variables físicoquímicas analizadas en el río Oma a lo largo del periodo de estudio (valores en $\mathrm{mg} / \mathrm{l}$ excepto para la dureza, en grados Franceses, y la conductividad, en $\mu \mathrm{S} / \mathrm{cm}$, en la figura $2 \mathrm{~A}$ ). Temporal variation of some of the physical and chemical variables measured in the Oma River during the study period (values in $\mathrm{mg} / \mathrm{l}$ except for the hardness, in French degrees, and conductivity, in $\mu \mathrm{S} / \mathrm{cm}$, in the figure $2 \mathrm{~A}$ ).

Si se consideran las campañas por separado se observan importantes diferencias en la composición faunística. En las campañas 94 y 95 una decena de familias presentan valores de dominancia superiores o iguales a un $1 \%$ y la composición faunística resulta ser similar: gasterópodos de la familia Hydrobiidae, básicamente Potamopyrgus, suponen algo menos de la mitad de los ejemplares mientras que otros taxones de abundancia relativa importante serían los baétidos, quironómidos y élmidos. A partir de 1996 la dominancia de Hydrobiidae comienza a ser muy acusada hasta alcanzar casi el $80 \%$ de los individuos en la campaña del 98 . En esas tres campañas sólo 6 ó 7 familias presentaban una dominancia $\geq 1 \%$. En la campaña de 2000 la

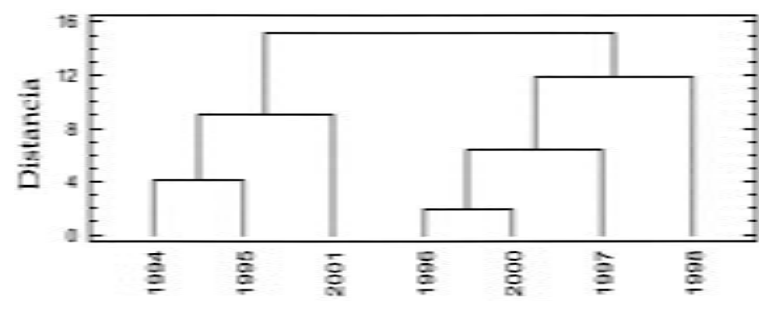

Figura 3. Dendrograma de afinidad entre campañas. Análisis de agrupamiento partir de las variables fisicoquímicas (coeficiente de distancia euclídea y algoritmo de agrupamiento de Ward). Dendrogram of affinity between sampling seasons. Cluster analysis based on the physical and chemical variables (Euclidean distance and agglomeration by Ward's method).

composición faunística es completamente distinta, encontrando hasta 13 familias con dominancia $\geq 1 \%$. Los Hydrobiidae no suponen ni un $5 \%$ del total, mientras que los efemerópteros representan altos porcentajes: Baetidae $(25.3 \%)$ y Ephemerellidae (12.5\%), al igual que los coleópteros de la familia Helodidae (16.2\%), de escasa importancia relativa en otras campañas. Finalmente, la composición faunística en el 2001 se asemeja más a las de campañas intermedias, aunque en esta ocasión los Ephemerellidae continúan suponiendo, como en el 2000, un importante porcentaje.

En las dos primeras campañas el número total de individuos por unidad de muestreo fue muy elevado (Tabla 2), pero a partir de 1995 se observa un fuerte descenso, con un mínimo de aproximadamente un millar de ejemplares, en las campañas 96 y 97. Aunque la abundancia aumenta en los años siguientes, nunca se alcanzan los valores del inicio del estudio. La riqueza, evaluada como el número de familias por campaña, oscila entre 26 y 38, observándose un patrón de variación similar al del índice IBMWP (Tabla 2); este índice siempre presenta valores muy elevados, por lo que el tramo se clasifica como de aguas muy limpias, de buena calidad. Sin embargo, la diversidad de Shannon sufre un descenso continuado desde 1994 hasta 1998, seguido de un fuerte incremento en el 2000, época en la que un mayor número de taxones presentaba valores de dominancia relativamente importantes. 
Tabla 2. Número de ejemplares de las muestras de macroinvertebrados bentónicos recogidas en el río Oma a lo largo del periodo de estudio. Número total de ejemplares y familias por campaña y valores de los índices de diversidad de Shannon y biótico (IBMWP). Number of individuals of the benthic macroinvertebrate samples collected in the Oma River during the study period. Total number of benthic macroinvertebrate individuals and families and Shannon diversity and biotic indexes (IBMWP).

\begin{tabular}{|c|c|c|c|c|c|c|c|c|c|}
\hline & TAXón & Código & $\begin{array}{l}30 / 06 \\
1994\end{array}$ & $\begin{array}{c}30 / 06 \\
1995\end{array}$ & $\begin{array}{c}24 / 06 \\
1996\end{array}$ & $\begin{array}{c}30 / 06 \\
1997\end{array}$ & $\begin{array}{l}24 / 06 \\
1998\end{array}$ & $\begin{array}{l}17 / 07 \\
2000\end{array}$ & $\begin{array}{l}18 / 07 \\
2001\end{array}$ \\
\hline \multirow[t]{2}{*}{ Turbellaria } & Planariidae & Planariidae & 6 & 9 & - & - & - & 2 & - \\
\hline & Dugesiidae & Dugesiidae & - & - & 7 & 14 & 4 & - & 52 \\
\hline \multirow[t]{3}{*}{ Annelida } & Oligochaeta & AOLI & 36 & 82 & 75 & 30 & 12 & 103 & 8 \\
\hline & Glossiphoniidae & AGLO & - & 6 & 7 & - & 1 & 1 & 18 \\
\hline & Hirudidae & AHIR & - & - & - & 1 & 2 & - & - \\
\hline \multirow[t]{4}{*}{ Mollusca } & Hydrobiidae & MHYD & 3587 & 3264 & 807 & 700 & 2613 & 82 & 2051 \\
\hline & Ancylidae & MANC & 28 & 71 & 6 & - & 8 & 6 & 4 \\
\hline & Planorbidae & MPLA & - & - & - & - & 1 & - & - \\
\hline & Sphaeriidae & MSPH & - & 3 & 1 & - & 1 & - & 1 \\
\hline Acari & Hydracarina & Hydracarina & 91 & 58 & 10 & 1 & 14 & - & - \\
\hline Crustacea & Gammaridae & gammaridae & 216 & 224 & 1 & 2 & 21 & 25 & 127 \\
\hline \multirow[t]{6}{*}{ Ephemeroptera } & Ephemeridae & ЕEPH & 3 & 1 & 4 & 1 & - & - & 2 \\
\hline & Caenidae & ECAE & - & - & 4 & - & - & - & - \\
\hline & Heptageniidae & EHEP & 13 & 18 & - & 2 & 1 & - & 7 \\
\hline & Baetidae & EBAE & 1584 & 1302 & - & 109 & 43 & 438 & 158 \\
\hline & Ephemerellidae & EEPHL & 357 & 114 & 36 & 15 & 87 & 217 & 424 \\
\hline & Leptophlebiidae & ELEP & 231 & 53 & 4 & 7 & 30 & - & 1 \\
\hline \multirow[t]{2}{*}{ Plecoptera } & Leuctridae & PLEU & 34 & 45 & 5 & 4 & 10 & 22 & 5 \\
\hline & Perlidae & PPER & 16 & 24 & - & - & 3 & 4 & - \\
\hline \multirow[t]{3}{*}{ Odonata } & Gomphidae & OGOM & - & - & 10 & 3 & - & 5 & - \\
\hline & Aeschnidae & OAES & - & - & - & 5 & 5 & 19 & 4 \\
\hline & Cordulegasteridae & OCOR & - & - & - & - & 1 & - & - \\
\hline Heteroptera & Gerridae & HGER & - & 26 & 3 & - & - & - & - \\
\hline \multirow[t]{5}{*}{ Coleoptera } & Dytiscidae & CDYT & - & - & 1 & 1 & 1 & - & - \\
\hline & Haliplidae & CHAL & - & - & - & - & 1 & - & 2 \\
\hline & Helodidae & CHEL & 27 & - & 12 & - & 5 & 280 & 13 \\
\hline & Dryopidae & CDRY & - & 24 & - & - & - & - & 4 \\
\hline & Elmidae & CELM & 906 & 435 & 120 & 25 & 99 & 118 & 142 \\
\hline \multirow[t]{12}{*}{ Trichoptera } & Rhyacophilidae & TRHY & 13 & 58 & 1 & - & 15 & 57 & 19 \\
\hline & Glossosomatidae & TGLO & - & 11 & - & - & - & - & - \\
\hline & Hydroptilidae & THYDT & 313 & 190 & 3 & 4 & 40 & 4 & 2 \\
\hline & Hydropsichydae & THYDS & 13 & 24 & 2 & - & 2 & 199 & 8 \\
\hline & Polycentropodidae & TPOL & 97 & 7 & - & 1 & 15 & - & - \\
\hline & Psychomyiidae & TPSY & 163 & 12 & 58 & 4 & 50 & - & 1 \\
\hline & Ecnomidae & TECN & - & 62 & - & - & - & - & - \\
\hline & Limnephilidae & TLIM & - & 3 & 1 & - & 11 & - & 13 \\
\hline & Goeridae & TGOE & 9 & 87 & 3 & - & 2 & 2 & 9 \\
\hline & Leptoceridae & TLEP & 30 & - & 7 & - & - & - & - \\
\hline & Odontoceridae & TODO & - & - & 1 & 1 & - & 2 & - \\
\hline & Beraeidae & TBER & 324 & - & - & - & 1 & - & - \\
\hline \multirow[t]{8}{*}{ Diptera } & Psychodidae & DPSY & - & 7 & - & 1 & - & - & - \\
\hline & Limoniidae & DLIM & 78 & 3 & 1 & - & 10 & 6 & 23 \\
\hline & Dixidae & DDIX & - & - & - & - & 1 & - & - \\
\hline & Simuliidae & DSIM & 6 & 26 & 3 & 1 & 2 & 31 & 6 \\
\hline & Chironomidae & DCHI & 1589 & 646 & 79 & 102 & 173 & 103 & 50 \\
\hline & Ceratopogonidae & DCER & 12 & 13 & 3 & - & - & - & - \\
\hline & Stratiomyidae & DSTR & - & 1 & 1 & - & 1 & 1 & - \\
\hline & Empididae & DEMP & 25 & 7 & 1 & 1 & - & 1 & 4 \\
\hline
\end{tabular}


Tabla 2. (Continuación.) (Continuation.)

\begin{tabular}{|c|c|c|c|c|c|c|c|c|c|}
\hline \multirow{8}{*}{ Megaloptera } & Dolichopodidae & DDOL & - & 3 & - & 1 & - & 4 & - \\
\hline & Tabanidae & DTAB & - & - & - & - & 1 & 1 & 2 \\
\hline & Anthomyiidae & DANT & - & - & - & 1 & 1 & - & - \\
\hline & Sialidae & Sialidae & - & 4 & - & - & 2 & - & 1 \\
\hline & & ABUNDANCIA & 6541 & 6923 & 1277 & 1037 & 3290 & 1733 & 3161 \\
\hline & & $\mathrm{N}^{\mathrm{o}}$ FAMILIAS & 28 & 36 & 32 & 26 & 38 & 26 & 30 \\
\hline & Divi & IDAD SHANNON & 2.97 & 2.73 & 2.21 & 1.85 & 1.50 & 3.32 & 2.00 \\
\hline & & IBMWP & 170 & 192 & 174 & 145 & 204 & 138 & 163 \\
\hline
\end{tabular}

La ordenación de taxones y campañas en el plano definido por los 2 primeros ejes factoriales de correspondencias se representa en la figura 4. Las campañas 1994 y 1995 quedan claramente diferenciadas entre sí y respecto a años siguientes, lo mismo que las campañas 96 a 98 de las dos últimas 2000-01. Hay una serie de taxones que, aunque de presencia más o menos constante a lo largo del periodo de estudio, son más abundantes en las dos primeras campañas (Hydracarina, Leptophlebiidae, Hidroptilidae, Chironomidae y Empididae). Por el contrario, no se recogen larvas de odonatos, que sí aparecen en las muestras a partir de 1996. Hay otras familias que, teniendo abundancias importantes en el periodo 94-95, posteriormente desaparecen o se hacen muy escasas en número de ejemplares, pero vuelven a aparecer al final del estudio: así, en la campaña 2000 (Baetidae), en la de 2001 (Gammaridae), o en las dos (Ephemerellidae, Elmidae). Finalmente, algunas familias son exclusivas de una o dos campañas, como Dryopidae y Ecnomidae en la campaña 95, o bien son recogidas en más campañas pero con abundancia elevada sólo en una de ellas, como Goeridae en el 95, o Helodidae en el 2000.

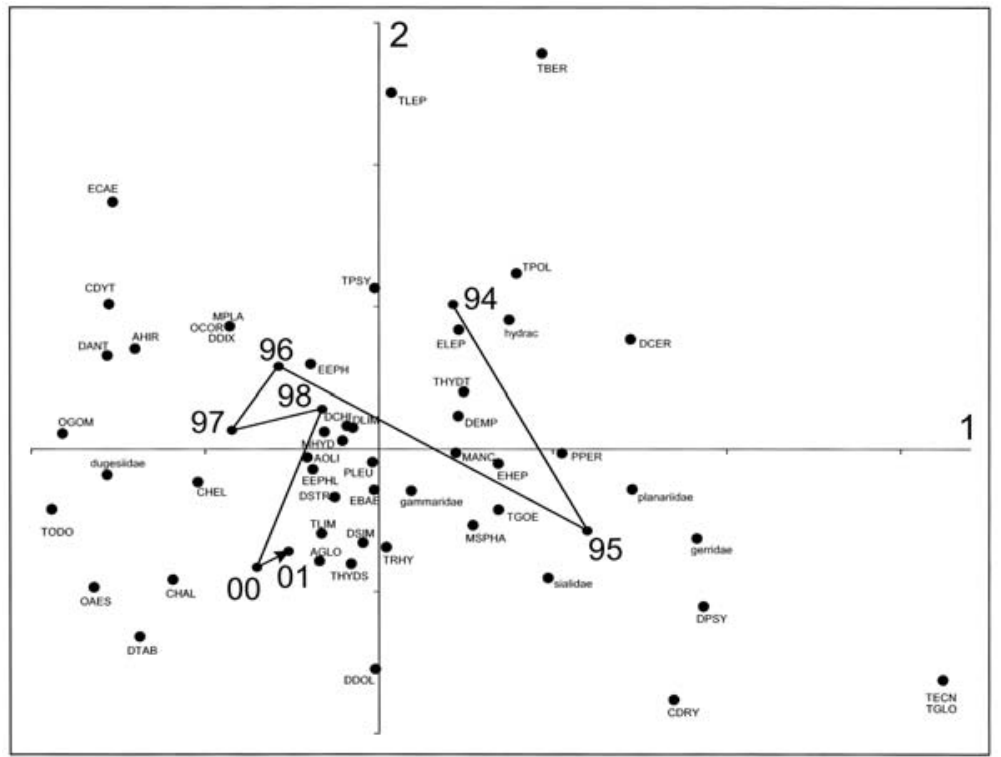

Figura 4. Localización de las familias y campañas en el plano definido por los 2 primeros ejes del análisis factorial de correspondencias. Cada familia está codificada por 4 o 5 letras mayúsculas (ver Tabla 2), correspondiendo la primera de ellas a una categoría taxonómica superior $(\mathrm{A}=$ Anélidos, $\mathrm{M}=$ Moluscos, $\mathrm{E}=$ Efemerópteros, $\mathrm{P}=$ Plecópteros, $\mathrm{O}=\mathrm{Odonatos}, \mathrm{C}=\mathrm{Coleópteros}, \mathrm{T}=\mathrm{Tricópteros}, \mathrm{D}=$ Dípteros). Location of the families and sampling years in the plane defined by the two first axes of the factorial correspondence analysis. The first of the 4 or 5 capital letters that identify each family (see table 3), belong to a higher taxonomical level (A= Annelida, $M=$ Mollusca, $E=$ Ephemeroptera, $P=$ Plecoptera, $O=$ Odonata, $C=$ Coleoptera, $T=$ Trichoptera, $D=$ Diptera). 


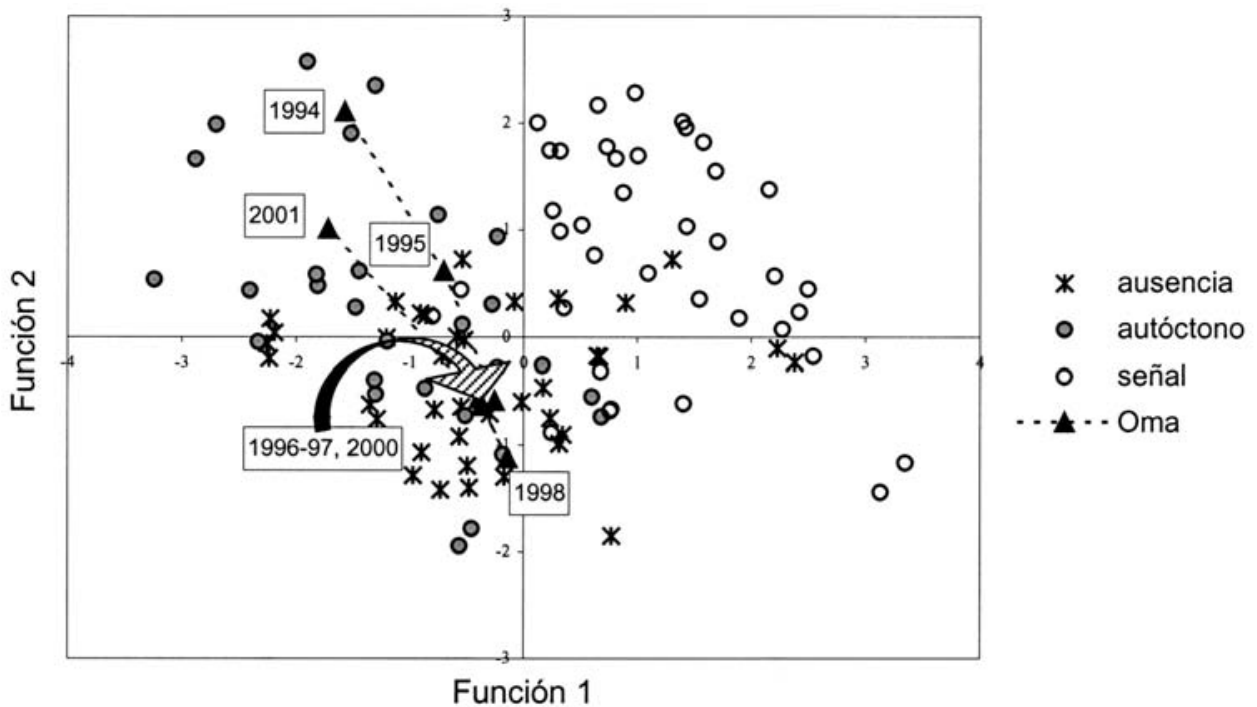

Figura 5. Análisis discriminante según la química del agua y ordenación de los casos con presencia o ausencia de las diferentes especies de cangrejo. Se señalan las muestras correspondientes al río Oma. Discriminant analysis based on water chemistry and cases ordination with presence or absence of different species of crayfish. The samples from the Oma River are indicated.

La interpretación de los ejes factoriales de correspondencias se hace estableciendo la correlación de cada uno de ellos con las variables físicoquímicas mediante el índice $\mathrm{r}$ de Pearson. Se demuestran las siguientes relaciones estadísticamente significativas $(n=7$; $\mathrm{p}<0.05 ; * \mathrm{p}<0.01 ;$ n.s. $=$ no significación):

\begin{tabular}{ccc}
\hline & + & - \\
\hline FAC1 & $\mathrm{pH} \mathrm{SO}_{4}^{-2}$, Sólidos suspensión* & $\mathrm{SiO}_{2}$ \\
FAC2 & $\mathrm{Mg}^{+2}$, Sólidos en disolución* & Oxígeno* \\
FAC3 & n.s. & n.s. \\
\hline
\end{tabular}

Por último, se aplicaron las ecuaciones obtenidas en el modelo de distribución de las especies de cangrejo según las condiciones fisicoquímicas del agua (Rallo \& García-Arberas, 2002) a los datos de las dos últimas campañas (Fig. 5). Según este modelo, la muestra del 2000 quedaría clasificada como "sin cangrejos" mientras que en la campaña 2001 el río Oma, según la química del agua, se clasificaría como apto para ser habitado por cangrejo autóctono.

\section{DISCUSIÓN}

La población de cangrejo autóctono habitante en el río Oma, bien establecida por décadas, se extinguió entre los veranos de los años 1994 y 1995. También se han encontrado cambios en las condiciones abióticas y bióticas del río, suficientes como para que sus aguas sean clasificadas desde los años 95 a 2000 como "no aptas" para la vida del cangrejo autóctono. ¿Cuál, o cuáles pueden haber sido las causas de estos cambios, y en particular de la desaparición del cangrejo en el Oma?

Aunque la afanomicosis continúa actualmente originando desapariciones de poblaciones nativas de cangrejo autóctono en la Península Ibérica (Dieguez-Uribeondo et al., 1997b), en el Oma no se observaron mortandades masivas (comunicación de la guardería). Además un episodio catastrófico de este tipo no originaría un cambio inmediato en las condiciones del hábitat, que habría quedado como "nicho vacío". Por todo ello se considera como muy poco probable la desaparición por peste o por otras enfermedades causantes de episodios catastróficos. 
La presión excesiva de depredadores también puede ser motivo de desaparición de poblaciones de cangrejos nativos, tanto directamente (Dieguez-Uribeondo et al., 1997a) como indirectamente, al interaccionar con especies de cangrejos introducidas (Söderbäck, 1994). La trucha es un depredador de A. pallipes (Foster \& Slater, 1990), pero este tramo del Oma había venido siendo repoblado tradicionalmente sin que ello supusiera una perturbación importante para el cangrejo. Respecto al furtivismo, aunque fuerte hacía algunos años, había pasado a ser prácticamente nulo en la zona (comunicación de la guardería). Por otra parte, esta presión afectaría en principio a determinadas clases de edad, de forma que la desaparición de la población habría sido de forma gradual. Tampoco es, por tanto, aceptable esta propuesta como causa de la extinción.

Lo que sí está demostrado es que en las mismas fechas de la desaparición del cangrejo ocurrieron cambios en la condición fisicoquímica del río. En el valle del Oma (sin industria, con densidad de población baja y usos agrícola y ganadero) el ganado accede en algunas zonas al cauce, lo que explicaría el valor inusualmente alto de $\mathrm{NH}_{4}{ }^{+}$en la campaña 1994, pero no parece el motivo de la desaparición de la población de $A$. pallipes. En otras zonas de la red fluvial de Bizkaia se han confirmado mortandades masivas de ejemplares de cangrejo señal por el uso inadecuado de biocidas de uso agrícola (Rallo et al., 2000), por lo que no podemos descartar que lo ocurrido en el Oma, en donde también existen huertas cercanas al río, hubiera podido deberse a un vertido de estos productos, puntual o continuado durante un período, que también podrían haber afectado a otros taxones (como otros crustáceos o insectos). En este valle también existen explotaciones madereras por lo que es probable el aporte de sólidos por deforestación de la cuenca. De hecho el primer factor de correspondencias está vinculado al incremento de la concentración de sólidos en suspensión, y el macrobentos sí podría haberse visto afectado. Por otra parte, las fumigaciones forestales con DIMILIN® (diflubenzuron) se vienen realizando con una cierta frecuencia sobre pinares en la provin- cia y es probable, aunque no comprobado, que se hayan podido dar lixiviados de insecticida.

También se ha considerado el hecho de que en cabecera de la cuenca existe un vertedero en donde se depositaron durante unos 20 años residuos sólidos urbanos. Aunque se clausuró en 1987 y fue parcialmente sellado un año después, se constató la existencia de lixiviados que afectaban su entorno inmediato (Morales et al., 1994). No obstante, el efecto se veía amortiguado por dilución, de forma que ya aguas arriba del barrio de Oma estos autores no detectaban concentraciones anómalas de ninguna de las variables en las fechas de su trabajo. Pero puede que las haya habido después.

Las intervenciones en cauce pueden ser también motivo de mortandades de cangrejos, lo que se ha constatado en otras zonas de la red fluvial de la provincia (Rallo et al., 1996; Rallo et al., 2001). En el caso del Oma, no tenemos constancia de que se realizara ninguna intervención ni en el cauce ni en zonas cercanas al río durante el periodo de estudio.

También hay que considerar la posibilidad de que sea la desaparición de los cangrejos, debida a causas no determinadas, la que provoque las modificaciones encontradas en el río casi simultáneamente y después. Está demostrado que los cangrejos influyen en la estructura de la comunidad bentónica, tanto por consumo directo de macroinvertebrados como indirectamente, al modificar el hábitat: por ejemplo, destruyendo cobijos al consumir macroalgas y macrófitos (Matthews et al., 1993; Momot, 1995; Guan \& Wiles, 1998; Nyström, 1999). Esta influencia se ha podido evaluar en zonas en donde han desaparecido poblaciones autóctonas (Matthews \& Reynolds, 1992) o, por el contrario, en ríos y lagos en donde se han realizado introducciones de especies alóctonas (Nyström et al., 1996, 1999). Aunque a corto plazo la influencia de los cangrejos en los niveles tróficos va a depender de su eficacia en la alimentación, de las preferencias alimenticias y de las tasas de consumo específicas según las especies, en general tienen un impacto negativo sobre la densidad de moluscos de pequeña talla y concha fina mientras que 
los taxones más activos, como coleópteros, heterópteros y gammáridos se verían menos afectados. En el Oma a partir de 1996 también se apreciaron cambios importantes en la composición, abundancia y diversidad faunística del macrobentos, sin que el índice biótico IBMWP presentara gran variación. Algunos de estos cambios podrían estar relacionados con la desaparición de los cangrejos. Así, los baétidos son muy abundantes en las dos primeras campañas pero no se recogen en el 1996, y algunos trabajos de campo muestran que este grupo aparece afectado positivamente por la población de cangrejos (Nyström et al., 1999). No obstante, encontramos resultados aparentemente contradictorios y, por ejemplo, no se registra un incremento en la abundancia de macroinvertebrados como cabría esperar al desaparecer el depredador. Otros taxones también desaparecen o se presentan en densidades muy bajas a partir del 95, aunque existen diversos factores que pueden influir, como por ejemplo la depredación por truchas en el caso de los gammáridos (Dahl, 1998).

A partir del 95 también se observó un incremento de macrófitos fluviales. En otras zonas la densidad de macrófitos se ha encontrado negativamente relacionada con la de cangrejos (Matthews et al., 1993; Nyström \& Strand, 1996), aunque existe controversia sobre la importancia de la fracción vegetal en la dieta de los cangrejos y parece ser que ésta varía según las especies (Goddard, 1988; Guan \& Wiles, 1998). En todo caso el incremento inicial de macrófitos tiene un efecto sobre el hidrodinamismo aumentando el depósito de sólidos y favoreciendo por tanto el asentamiento y crecimiento de nuevos macrófitos. Todo ello obviamente también provoca modificaciones en la comunidad de macroinvertebrados.

En conclusión, y aunque en otras cuencas cercanas hemos podido detectar la causa principal de mortandad y/o desaparición de cangrejos, no es así en el Oma, en donde podrían haber actuado sinérgicamente varios factores. No obstante, la evolución temporal del sistema río en los últimos años parece presentar una tendencia a recuperar las condiciones que tenía en el año
1994, tanto en la fisicoquímica como en la composición faunística. De hecho, siguiendo el modelo de condición de hábitat para el cangrejo según la química del agua (Rallo \& GarcíaArberas, 2002), el tramo estudiado en la campaña 2001 sería apto para acoger cangrejo autóctono. En este sentido se ha aconsejado a la administración la reintroducción de la especie y su seguimiento de forma continuada.

\section{AGRADECIMIENTOS}

Este trabajo ha sido realizado en colaboración con el Dpto. de Agricultura de la Diputación Foral de Bizkaia.

\section{BIBLIOGRAFÍA}

ALBA-TERCEDOR, J. \& A. SANCHEZ-ORTEGA. 1988. Un método rápido y simple para evaluar la calidad biológica de las aguas corrientes basado en el de Hellawell (1978). Limnetica, 4: 51-56.

APHA-AWWA-WPCF. 1998. Standard methods for the examination of water and wastewater. $20^{\text {th }} \mathrm{ed}$. L.S. Clesceri, A.E. Greenberg \& A.D. Eaton (eds.), Washington DC.

DAHL, J. 1998. The impact of vertebrate and invertebrate predators on a stream benthic community. Oecologia, 117: 217-226.

DIEGUEZ-URIBEONDO, J., A. RUEDA, E. CASTIEN \& J. C. BASCONES, 1997a. A plan of restoration in Navarra for the native freshwater crayfish species of Spain, Austropotamobius pallipes. Bull. Fr. Pêche Piscic.,347: 625-637.

DIEGUEZ-URIBEONDOJ., C. TEMIÑO, \& J. L. MUZQUIZ. 1997b The crayfish plague fungus (Aphanomyces astaci) in Spain. Bull. Fr. Pêche Piscic., 347: 753-763.

FOSTER, J. \& F. M. SLATER, 1990. A global review of crayfish predation with observation of the possible loss of Austropotamobius pallipes in the Welsh Wye due to crayfish plague. Freshw. Crayfish, 8: 589-613.

GARCÍA-ARBERAS, L. \& A. RALLO. 2000. Survival of natural populations of Austropotamobius pallipes in rivers in Biscay, Basque Country (North of Iberian Peninsula). Bull. Fr. Pêche Piscic., 356: 17-30. 
GODDARD, J. S. 1988. Food and feeding. In: Freshwater crayfish. Biology, Management and exploitation. D.M. Holdich \& R.S. Lowery (eds.): 145-166. The University Press, Cambridge.

GUAN, R. Z. \& P. R. WILES. 1998. Feeding ecology of the signal crayfish Pacifastacus leniusculus in a British lowland river. Aquaculture, 169: 177-193.

MANUGISTICS INC., 1997. Statgraphics Plus for Windows (vers. 4.1). Rockville, MD, USA.

MATTHEWS, M. A. \& J. D. REYNOLDS. 1992. Ecological impact of crayfish plague in Ireland. Hydrobiologia, 234: 1-6.

MATTHEWS, M. A., J. D. REYNOLDS \& M. J. KEATINGE. 1993. Macrophyte reduction and benthic community alteration by the crayfish Austropotamobius pallipes (Lereboullet). Freshw. Crayfish, 9: 289-299.

MOMOT, W. T. 1995. Redefining the role of crayfish in aquatic ecosystems. Rev. Fish. Sci., 3: 33-63.

MORALES, T., M. A. GÓMEZ, I. ANTIGÜEDAD, R. ZUBIAGA, A. BARCENILLA \& A. BONILLA. 1994. Reconocimiento del impacto en aguas subterráneas de dos vertederos clausurados en la Reserva de la Biosfera de la comarca de Urdaibai (País Vasco). Actas Congreso Análisis y evolución de la contaminación de las aguas subterráneas. Alcalá de Henares, España, T.II: 309-324.

NYSTRÖM, P. 1999. Ecological impact of introduced and native crayfish on freshwater communities: European perspectives. In: Crayfish in Europe as alien species. How to make the best of a bad situation?. F. Gherardi \& D.M. Holdich (eds.): 63-85. A.A. Balkema, Rotterdam. Netherlands.

NYSTRÖM, P., C. BRÖNMARK \& W. GRANÉLI. 1996. Patterns in benthic food webs: a role for omnivorous crayfish? Freshw. Biol., 36: 631-646.

NYSTRÖM, P., C. BRÖNMARK \& W. GRANÉLI. 1999. Influence of an exotic and a native crayfish species on a littoral benthic community. Oikos, 85: 545-553.
NYSTRÖM, P. \& J. A. STRAND, 1996. Grazing by a native and an exotic crayfish on aquatic macrophytes. Freshw. Biol., 36: 673-682

RALLO, A., L. GARCÍA-ARBERAS \& A. ANTÓN. 2000. Informe sobre la mortandad de cangrejo señal en el río Aretxabalagane, aguas abajo de Goikolejea, en setiembre de 2000. Informe técnico, Diputación Foral de Bizkaia, 10 pp.

RALLO, A., L. GARCÍA-ARBERAS \& A. ANTÓN. 2001. Relationships between changes in habitat conditions and population density of an introduced population of signal crayfish (Pacifastacus leniusculus) in a fluvial system. Bull. Fr. Pêche Piscic., 361: 643-657.

RALLO, A. \& L. GARCÍA-ARBERAS. 2002. Differences in abiotic water conditions between fluvial reaches and crayfish fauna in some northern rivers of the Iberian Peninsula. Aquat. Living. Resour., 15: 119-128.

RALLO, A., T. SERRANO, G. FERRERO, A. ANTÓN, L. GARCÍA-ARBERAS \& A. ECHEANDIA. 1996. Informe sobre la mortandad de cangrejo señal ocurrida en el río Aretxabagane desde el núcleo urbano de Larrabetxu hasta unos $4 \mathrm{~km}$ aguas abajo en mayo de 1996. Informe técnico, Diputación Foral de Bizkaia, 21 pp.

RICO E., A. RALLO, M. SEVILLANO \& M. L. ARRETXE. 1992. Comparison of several biological indices based on river macroinvertebrate benthic community for assessment of running water quality. Ann. Limnol.-Int. J. Limnol., 28: 147-156.

ROHLF, F. J. 1997. NTSYSpc. Numerical taxonomy and multivariate analysis system ver. 2.0. Exeter Software, New York.

SÖDERBÄCK, B. 1994. Interactions among juveniles of two freshwater crayfish species and a predatory fish. Oecologia, 100: 229-235.

SÖDERHÄLL, K. \& L. CERENIUS. 1999. The crayfish plague fungus: history and recent advances. Freshw. Crayfish, 12. 11-35. 
